\title{
SET-VALUED STOCHASTIC LEBESGUE INTEGRAL AND REPRESENTATION THEOREMS
}

\author{
JUNGANG LI \\ Department of Applied Mathematics,Beijing University of Technology \\ 100 Pingleyuan, Chaoyang District, Beijing, 100022, P.R.China \\ E-mail: ljg001@emails.bjut.edu.cn \\ SHOUMEI LI \\ Department of Applied Mathematics,Beijing University of Technology \\ 100 Pingleyuan, Chaoyang District, Beijing, 100022, P.R.China \\ E-mail:lisma@bjut.edu.cn \\ Received:26-09-2007 \\ Revised:19-12-2007
}

\begin{abstract}
In this paper, we shall firstly illustrate why we should introduce set-valued stochastic integrals, and then we shall discuss some properties of set-valued stochastic processes and the relation between a set-valued stochastic process and its selection set. After recalling the Aumann type definition of stochastic integral, we shall introduce a new definition of Lebesgue integral of a set-valued stochastic process with respect to the time $t$. Finally we shall prove the presentation theorem of set-valued stochastic integral and discuss further properties that will be useful to study set-valued stochastic differential equations with their applications.
\end{abstract}

Keywords: set-valued stochastic process, selection process, set-valued Lebesgue integral, representation theorem.

\section{Introduction}

It is well-known that classical stochastic differential equations have widely been used in optimal control problems ${ }^{1}$, mathematical finance ${ }^{2,3}$ and so on. Since the dynamical systems concerning practical uses are complex, the dynamical systems having velocities are usually not determined uniquely by the state of the systems. The investigations of this kind systems led to replacement of the differential equation $\dot{x}(t)=f(t, x(t))$ with the differential inclusion $\dot{x}(t) \in F(t, x(t))$, where $F$ is a set-valued function. This kind of situation appears in studying the evolution of macro-systems in economic, social or biological sciences, where very often it is difficult to determine velocities uniquely.

On the other hand, we have to consider the systems in which there are random disturbances in the real world. In this case, some stochastic optimal control problems can be described by setvalued stochastic differential inclusions. Indeed, assume that $f=\left\{\left(f_{t}(z, p)\right)_{t \in I}:(z, p) \in R^{d} \times U\right\}, g=$ $\left\{\left(g_{t}(z, p)\right)_{t \in I}:(z, p) \in R^{d} \times U\right\}$ are $d$-dimensional measurable and adapted stochastic processes depending on parameters $z \in R^{d}, p \in U$, where $U$ is a fixed set and $I$ is the set of time, for examples, $I=[0, T]$ or $I=[0, \infty)$, then the control equation is

$$
x_{t}=\xi+\int_{0}^{t} f_{s}\left(x_{s}, u_{s}\right) d s+\int_{0}^{t} g_{s}\left(x_{s}, v_{s}\right) d B_{s},
$$

for any $t \in I$ a.e., where $B=\left(B_{t}\right)_{t \in I}$ is a Brownian motion. The stochastic process $\left(\left(u_{t}\right)_{t \in I}, \quad\left(v_{t}\right)_{t \in I}\right)$ is called a strategy or a control taking values in $U$. If $\mathcal{C}, \mathcal{U}, \mathcal{V}$ are given sets of constraints and strategies respectively, we shall look for the triples 
$\left(x^{u, v}, u, v\right)$ such that $x^{u, v}=\left(x_{t}^{u, v}\right)_{t \in I}$ is the solution of (1.1), and $\left(x^{u, v}, u, v\right) \in \mathcal{C} \times \mathcal{U} \times \mathcal{V}$. If for any fixed $t \in I, z \in R^{d}$, put $F_{t}(z)=\left\{f_{t}\left(z, u_{t}\right): u \in \mathcal{U}\right\}$; $G_{t}(z)=\left\{g_{t}\left(z, v_{t}\right): v \in \mathcal{V}\right\}$. Then to look for the solutions of (1.1) becomes to determine the solution set of the following set-valued stochastic differential inclusion:

$$
d x_{t} \in F_{t}\left(x_{t}\right) d t+G_{t}\left(x_{t}\right) d B_{t}, \quad x_{0}=\xi
$$

or stochastic integral form

$$
x_{t}-x_{s} \in \operatorname{cl}\left(\int_{s}^{t} F_{\tau}\left(x_{\tau}\right) d t+G_{\tau}\left(x_{\tau}\right) d B_{\tau}\right), \quad s, t \in I
$$

The purpose of stochastic optimal control is to minimize the expectation value of a given function $c$ : $\mathcal{C} \times \mathcal{U} \times \mathcal{V} \rightarrow R$, where $c$ characterizes the cost of the loss or the errors related to the choice of a given control strategy and $\bar{c}(u, v)=E\left[c\left(x^{u, v}, u, v\right)\right]$ is called the cost of control.

In (1.2), there are two parts: one is the part $F_{t}\left(x_{t}\right) d t$ which is related to the integral of a setvalued stochastic process with respect to time $t$, i.e. $\int_{0}^{t} F_{s}\left(x_{s}\right) d s$, and the other is the part $G_{t}\left(x_{t}\right) d B_{t}$ which is related to the Ito integral of a set-valued stochastic process with respect to $B_{t}$. How to define these two integrals suitably is the first problem in the theory of set-valued stochastic analysis. What properties do they have? These problems are what we shall consider.

There are many good works in this area. In 1994, Ahmed ${ }^{4}$ introduced set-valued differential inclusion with the special case that the second part $G$ of (1.2) is a real-valued function. Kisielewicz ${ }^{5-10}$ discussed set-valued stochastic integral and solutions problems of general stochastic differential inclusion (1.2). In 1998, Aubin and Prato ${ }^{11}$ obtained a viability theorem for stochastic differential inclusion, and Motyl ${ }^{12}$ discussed stability problem for stochastic inclusion. We also would like to show our thanks to Polish mathematicians for their telling us the nice summary in this area 13,14 .

However, there are only a few papers to discuss stochastic integrals of set-valued stochastic process. Kim ${ }^{15}$ used the definition of stochastic integral of set-valued stochastic process introduced by Kisielewicz ${ }^{6}$ and discussed its properties. We called it Aumann type integral since the idea came from
Aumann integral of a set-valued random variable ${ }^{16}$. We may consider the concept of Ito integral of a setvalued stochastic process by another way, because the Ito integral of a set-valued stochastic process with respect to a Browniwn motion in $R^{d}$ should be a set-valued stochastic processes in $R^{d}$ rather than in $L^{2}\left[\Omega, R^{d}\right]$. So is the set-valued Lebesgue integral. Jung and $\mathrm{Kim}^{17}$ gave a new definition with basic space being $R$ by taking fixed time $t$. It is a quite nice work. But we still think that this new definition is necessary to correct again since it may be more suitable to treat a set-valued stochastic process as a whole. $\mathrm{Li}$ and $\mathrm{Ren}^{18}$ introduced a new way to define the Ito integral of set-valued stochastic processes and discussed their properties. In this paper, we should consider the Lebesgue integral of set-valued stochastic processes and their properties.

We organize our paper as following: in section 2 , we shall introduce some necessary notations, definitions and results about set-valued stochastic processes. In section 3, we shall give a new definition of Lebesgue integral of a set-valued process with respect to the time $t$. We shall also discuss some properties of set-valued stochastic integral, especially the presentation theorem of set-valued stochastic Lebesgue integral.

\section{Representation theorems of set-valued $L^{p}$ - bounded progressively measurable processes}

Throughout this paper, assume that $R$ is the set of all real numbers, $I=[0, T], N$ is the set of all natural numbers, $R^{d}$ is the $d$-dimensional Euclidean space with usual norm $\|\cdot\|, \mathcal{B}(E)$ is the Borel field of the space $E,(\Omega, \mathcal{A}, \mu)$ is a complete atomless probability space, the $\sigma$-field filtration $\left\{\mathcal{A}_{t}: t \in I\right\}$ satisfies the usual conditions (i.e. complete, non-decreasing and right continuous). Let $L^{p}\left[\Omega, \mathcal{A}_{t}, \mu ; R^{d}\right]$ be the set of $R^{d}$-valued $\mathcal{A}_{t}$-measurable random variables $\xi$ with the expectation $E\left[\|\xi\|^{p}\right]<\infty(p \geq 1)$. When $\mathcal{A}_{t}$ is replaced by $\mathcal{A}, L^{p}\left[\Omega, \mathcal{A}, \mu ; R^{d}\right]$ can be written as $L^{p}\left[\Omega, R^{d}\right]$

Let $f=\left\{f(t), \mathcal{A}_{t}: t \in I\right\}$ be a $R^{d}$-valued adaptive stochastic process. It is said that $f$ is progressively measurable if for any $t \in I$, the mapping $(s, \omega) \mapsto f(s, \omega)$ from $[0, t] \times \Omega$ to $R^{d}$ is $\mathcal{B}([0, t]) \times$ $\mathcal{A}_{t}$-measurable. If denote

$\left\{A \subset I \times \Omega: \forall t \in I, A \cap([0, t] \times \Omega) \in \mathcal{B}([0, t]) \times \mathcal{A}_{t}\right\}$, 
as $\mathcal{C}$, then $f$ is progressively measurable if and only if $f$ is $\mathrm{C}$-measurable. Each right continuous (left continuous) adaptive process is progressively measurable.

Assume that $\mathcal{L}^{p}\left(R^{d}\right)$ denotes the set of $R^{d}$-valued stochastic processes $f=\left\{f(t), \mathcal{A}_{t}: t \in I\right\}$ such that $f$ satisfying (a) $f$ is progressive; and (b)

$$
\|\| f \|_{p}=\left[E\left(\int_{0}^{T}\|f(t, \omega)\|^{p} d s\right)\right]^{1 / p}<\infty
$$

Let $f, f^{\prime} \in \mathcal{L}^{p}\left(R^{d}\right), f=f^{\prime}$ if and only if \|\|$f-$ $f^{\prime} \|_{p}=0$. Then $\left(\mathcal{L}^{p}\left(R^{d}\right),\|\| \cdot\|\|_{p}\right)$ is complete.

Now we review notation and concepts of setvalued stochastic processes.

Assume that $\mathbf{K}\left(R^{d}\right)$ is the family of all nonempty, closed subsets of $R^{d}$ with usual norm $\|\cdot\|$, and $\mathbf{K}_{c}\left(R^{d}\right)$ (resp. $\mathbf{K}_{k}\left(R^{d}\right), \mathbf{K}_{k c}\left(R^{d}\right)$ ) is the family of all nonempty closed convex (resp. compact, compact convex) subsets of $R^{d}$.

For any $x \in R^{d}, A$ is a nonempty subset of $R^{d}$, define the distance of $x$ and $A$

$$
d(x, A)=\inf _{y \in A}\|x-y\| .
$$

The Hausdorff metric on $\mathbf{K}\left(R^{d}\right)$ is defined as:

$$
d_{H}(A, B)=\max \left\{\sup _{a \in A} d(a, B), \sup _{b \in B} d(b, A)\right\}
$$

for $A, B \in \mathbf{K}\left(R^{d}\right)$. Note that the Hausdorff metric between two closed sets $A, B$ may take infinite when they are unbounded. But it is known ( Theorem 1.1.2 ${ }^{19}$ ) that the family of all bounded elements in $\mathbf{K}\left(R^{d}\right)$ is a complete separable space with respect to the Hausdorff metric $d_{H}$, and $\mathbf{K}_{k}\left(R^{d}\right)$ and $\mathbf{K}_{k c}\left(R^{d}\right)$ are its closed subsets. For $B \in \mathbf{K}\left(R^{d}\right)$, define $\|B\|_{\mathbf{K}}=d_{H}(\{0\}, B)=\sup _{a \in B}\|a\|$.

For a set-valued random variable $F,{ }^{19}{ }^{20}$ define the set

$$
S_{F}^{p}=\left\{f \in L^{p}\left[\Omega, R^{d}\right]: f(\omega) \in F(\omega) \text { a.e. }(\mu)\right\} .
$$

where $L^{p}\left[\Omega, R^{d}\right]$ is the set of all $R^{d}$-valued random variables $f$ such that $\|f\|_{p}=\left[E\left(\|f\|^{p}\right)\right]^{1 / p}<\infty$, and constant $p \geq 1$. The expectation of $F$ is defined as

$$
E[F]=\left\{E[f]: f \in S_{F}^{1}\right\}
$$

It is called the Aumann integral introduced by Aumann $^{16}$ in 1965 . A set-valued random variable $F: \Omega \rightarrow \mathbf{K}\left(R^{d}\right)$ is called integrable if $S_{F}^{1}$ is non-empty. $F$ is called integrable bounded if $\int_{\Omega}\|F(\omega)\|_{\mathbf{K}} d \mu<\infty$. Let $L^{p}\left[\Omega, \mathcal{A}, \mu ; \mathbf{K}\left(R^{d}\right)\right]$ (resp. $\left.L^{p}\left[\Omega, \mathcal{A}, \mu ; \mathbf{K}_{c}\left(R^{d}\right)\right], \quad L^{p}\left[\Omega, \mathcal{A}, \mu ; \mathbf{K}_{k c}\left(R^{d}\right)\right]\right)$ denote the family of $\mathbf{K}\left(R^{d}\right)$-valued (resp. $\mathbf{K}_{c}\left(R^{d}\right), \mathbf{K}_{k c}\left(R^{d}\right)$ valued) $L^{p}$-bounded random variables $F$ such that $\|F(\omega)\|_{\mathbf{K}} \in L^{p}[\Omega, R]$. Concerning more definitions and results of set-valued random variables, readers could refer to the book ${ }^{19}$ or the excellent paper. ${ }^{20}$

$F=\{F(t): t \in I\}$ is called a set-valued stochastic process if $F: I \times \Omega \rightarrow \mathbf{K}\left(R^{d}\right)$ is a set-valued function such that for any fixed $t \in I, F(t, \cdot)$ is a setvalued random variable.

A set-valued process $F=\{F(t): t \in I\}$ is called adapted with respect to the filtration $\left\{\mathcal{A}_{t}: t \in I\right\}$, if $F(t)$ is measurable with respect to $\mathcal{A}_{t}$ for each $t \in I$, and denoted by $\left\{F(t), \mathcal{A}_{t}: t \in I\right\} . F$ is called measurable if $F$ is $I \times \Omega$ measurable, i.e. $\{(t, \omega) \in I \times \Omega$ : $F(t, \omega) \cap A \neq \emptyset\} \in \mathcal{B}(I) \times \mathcal{A}$ for $A \in \mathcal{B}\left(R^{d}\right)$.

Definition 2.1 A set-valued stochastic process $F=$ $\{F(t): t \in I\}$ is called to be progressively measurable, if it is $\mathrm{C}$-measurable, i.e. for any $A \in$ $\mathcal{B}\left(R^{d}\right),\{(s, \omega) \in I \times \Omega: F(s, \omega) \cap A \neq \emptyset\} \in \mathcal{C}$.

If $F$ is progressively measurable then $F$ is adapted and measurable.

Definition 2.2 A progressively measurable setvalued stochastic process $F=\left\{F(t), \mathcal{A}_{t}: t \in I\right\}$ is called $\mathcal{L}^{p}$-bounded, if the real stochastic process $\left\{\|F(t)\|_{\mathbf{K}}, \mathcal{A}_{t}: t \in I\right\} \in \mathcal{L}^{p}(R)$.

Definition 2.3 A $R^{d}$-valued process $\left\{f(t), \mathcal{A}_{t}\right.$ : $t \in I\} \in \mathcal{L}^{p}\left(R^{d}\right)$ is called an $\mathcal{L}^{p}$-selection of $F=$ $\left\{F(t), \mathcal{A}_{t}: t \in I\right\}$ if $f(t, \omega) \in F(t, \omega)$ a.e. $(t, \omega) \in$ $I \times \Omega$.

Let $S^{p}(\{F(\cdot)\})$ or $S^{p}(F)$ denote the family of all $\mathcal{L}^{p}\left(R^{d}\right)$-selections of $F=\left\{F(t), \mathcal{A}_{t}: t \in I\right\}$, i.e.

$$
\begin{aligned}
S^{p}(F)=\{ & \{f(t)\} \in \mathcal{L}^{p}\left(R^{d}\right): f(t, \omega) \in F(t, \omega), \\
& \text { a.e. }(t, \omega) \in I \times \Omega\} .
\end{aligned}
$$

Please note the difference between $S^{p}(\{F(\cdot)\})$ and $S_{F(t)}^{p}\left(\mathcal{A}_{t}\right)$, the later means the selection set in $L^{p}\left[\Omega, \mathcal{A}_{t}, \mu ; R^{d}\right]$ of the set-valued random variable $F(t), t \in I$.

Theorem $2.4^{18,21}$ If $S^{p}(F) \neq \emptyset$, then $S^{p}(F)$ is a closed set of $\left(\mathcal{L}^{p}\left(R^{d}\right),\|\| \cdot\|\|_{p}\right)$. 
It is natural to ask under what conditions $S^{p}(F) \neq \emptyset$ and whether the set-valued stochastic process can be represented by a sequence in $S^{p}(F)$. The next theorem will answer these questions.

Theorem 2.5 ${ }^{19,21}$ Assume that $F=\{F(t): t \in I\}$ is an $\mathcal{L}^{p}$-bounded progressively measurable process. Then there exists a sequence of progressively measurable stochastic process $f_{n}: I \times \Omega \rightarrow R^{d}, n \geq 1$ in $S^{p}(F)$ such that for any $(t, \omega) \in I \times \Omega$,

$$
F(t, \omega)=\operatorname{cl}\left\{f_{n}(t, \omega): n \geq 1\right\} .
$$

Furthermore, if $F$ is an $\mathcal{L}^{p}$-bounded closed convex progressively measurable set-valued stochastic process and satisfies

$\left(C_{1}\right)$ for any $\omega \in \Omega, t \mapsto F(t, \omega)$ is lower semicontinuous (1.s.c.),

then there exists a sequence of Caratheodory selections $f_{n}: I \times \Omega, n \geq 1$ of $F$ such that for any $(t, \omega) \in I \times \Omega,(2.3)$ exists.

Remark 2.6 For the definition of 1.s.c. of a setvalued function, readers may refer to ${ }^{29}$ or ${ }^{21}$ ). A set-valued stochastic process is said to be trajectory lower semi-continuous (1.s.c.) if it satisfies $\left(C_{1}\right)$. Similarly, we may also have trajectory upper semi-continuous (u.s.c.), trajectory continuous, trajectory upper semi-continuous with respect to Hausdorff metric $d_{H}$ (h.u.s.c.), trajectory lower semicontinuous with respect to $d_{H}$ (h.l.s.c.) and trajectory continuous with respect to $d_{H}$.

Theorem 2.7 Assume that $F=\{F(t): t \in I\}$ is an $\mathcal{L}^{p}$-bounded progressively measurable set-valued stochastic process. Let $f_{n}: I \times \Omega \rightarrow R^{d}, n \geq 1$ be a sequence of progressively measurable stochastic processes in $S^{p}(F)$ such that for any $(t, \omega) \in I \times \Omega$,

$$
F(t, \omega)=\operatorname{cl}\left\{f_{n}(t, \omega): n \geq 1\right\} .
$$

Then, for any $f \in S^{p}(F)$ and $\varepsilon>0$, there exists a measurable partition $\left\{A_{1}, \cdots, A_{n}\right\}$ of $I \times \Omega$ such that

$$
\left\|\mid f-\sum_{i=1}^{n} I_{A_{i}} f_{i}\right\|_{p}<\varepsilon
$$

Proof Assume that for any $(t, \omega) \in I \times \Omega, f(t, \omega) \in$ $F(t, \omega)$ with $f \in S^{p}(F)$. Assume $\rho \in \mathcal{L}^{1}\left(R^{d}\right)$ is a positive stochastic process satisfying

$$
\int_{I \times \Omega} \rho(t, \omega) d(\lambda \times \mu)<\frac{\varepsilon^{p}}{3} .
$$

Let

$$
B_{1}=\left\{(t, \omega):\left\|f(t, \omega)-f_{1}(t, \omega)\right\|^{p}<\rho(t, \omega)\right\}
$$

Denote

$$
\left\{(t, \omega):\left\|f(t, \omega)-f_{n}(t, \omega)\right\|^{p}<\rho(t, \omega)\right\} \backslash\left(\cup_{i=1}^{n-1} B_{i}\right)
$$

as $B_{n}, n \geq 2$. Then $\left\{B_{i}\right\}$ is a countable measurable partition of $I \times \Omega$. Since $f, f_{1} \in \mathcal{L}^{p}\left(R^{d}\right)$, there exists an integer $n$ such that

$$
\sum_{i=n+1}^{\infty} \int_{B_{i}}\|f(t, \omega)\|^{p} d(\lambda \times \mu)<\frac{(\varepsilon / 2)^{p}}{3},
$$

and

$$
\sum_{i=n+1}^{\infty} \int_{B_{i}}\left\|f_{1}(t, \omega)\right\|^{p} d(\lambda \times \mu)<\frac{(\varepsilon / 2)^{p}}{3} .
$$

Let $A_{1}=B_{1} \bigcup\left(\bigcup_{i=n+1}^{\infty} B_{i}\right)$ and $A_{j}=B_{j}, 2 \leq j \leq n$, then

$$
\begin{aligned}
& \left\|\left|f-\sum_{i=1}^{n} I_{A_{i}} f_{i}\right|\right\|_{p}^{p} \\
& =E\left(\int_{0}^{T}\left\|f(s, \omega)-\sum_{i=1}^{n} I_{A_{i}} f_{i}(s, \omega)\right\|^{p} d s\right) \\
& \leq \sum_{i=1}^{n} \int_{B_{i}}\left\|f(s, \omega)-f_{i}(s, \omega)\right\|^{p} d(\lambda \times \mu) \\
& +\sum_{i=n+1}^{\infty} \int_{B_{i}}\left\|f(s, \omega)-f_{i}(s, \omega)\right\|^{p} d(\lambda \times \mu) \\
& \leq \int_{I \times \Omega} \rho(s, \omega) d(\lambda \times \mu)+\sum_{i=n+1}^{\infty} 2^{p} \int_{B_{i}}\left(\|f(s, \omega)\|^{p}\right. \\
& \left.+\left\|f_{1}(s, \omega)\right\|^{p}\right) d(\lambda \times \mu) \\
& <\varepsilon^{p} .
\end{aligned}
$$

Thus $\left\|\left|f-\sum_{i=1}^{n} I_{A_{i}} f_{i}\right|\right\|_{p}<\varepsilon$.

Theorem 2.8 Assume that $F=\{F(t): t \in I\}$ is a progressively measurable set-valued stochastic process, then $F$ is $\mathcal{L}^{p}$-bounded if and only if $S^{p}(F)$ is bounded in $\mathcal{L}^{p}\left(R^{d}\right)$.

Proof Assume that $F$ is $\mathcal{L}^{p}$-bounded, then $\bar{F}=$ $\left\{\|F(t)\|_{\mathbf{K}}, \mathcal{A}_{t}: t \in I\right\} \in \mathcal{L}^{p}(R)$. We have \|\|$f \|_{p} \leq$ $\||\bar{F}|\|_{p}<\infty$ for any $f \in S^{p}(F)$, i.e. $S^{p}(F)$ is bounded in $\mathcal{L}^{p}\left(R^{d}\right)$.

Now we consider opposite part. Assume that $S^{p}(F)$ is bounded in $\mathcal{L}^{p}\left(R^{d}\right)$. Since $F$ is a progressively measurable set-valued stochastic process, there exists a sequence of progressively measurable 
selections $f_{n} \in S^{p}(F), n \geq 1$ of $F$ such that for any $(t, \omega) \in I \times \Omega$,

$$
F(t, \omega)=\operatorname{cl}\left\{f_{n}(t, \omega): n \geq 1\right\},
$$

by Theorem 2.7. Thus by using Lemma $1.3 .12^{19}$ (or ${ }^{20}$ ) on product space $I \times \Omega$, we have

$E\left[\int_{0}^{T}\|F(s)\|_{\mathbf{K}}^{p} d s\right]=\sup _{f \in S^{p}(F)} E\left[\int_{0}^{T}\|f(s)\|^{p} d s\right]<\infty$,

Thus $F$ is $\mathcal{L}^{p}$-bounded.

Theorem 2.9 Assume that $F=\{F(t): t \in I\}$ is a progressively measurable closed convex setvalued stochastic process (i.e. for any $t \in I, \omega \in \Omega$, $\left.F(t, \omega) \in \mathbf{K}_{c}\left(R^{d}\right)\right)$, and $S^{p}(F) \neq \emptyset$, then $S^{p}(F)$ is convex.

Proof Let $\left\{f_{i}(t): t \in I\right\} \in S^{p}(F), i=1,2, a, b \geq 0$, and $a+b=1$. Since $F(t, \omega)$ is convex, for any $t \in I$, $\left(a f_{1}+b f_{2}\right)(t, \omega) \in F(t, \omega)$, a.e.. By Minkowski inequality on product space $I \times \Omega$, we have that

$$
\begin{aligned}
& \|\left\|a f_{1}+b f_{2} \mid\right\|_{p} \\
&= {\left[E\left(\int_{0}^{T}\left\|a f_{1}(s)+b f_{2}(s)\right\|^{p} d s\right)\right]^{1 / p} } \\
& \leq a\left[E\left(\int_{0}^{T}\left\|f_{1}(s)\right\|^{p} d s\right)\right]^{1 / p} \\
&+b\left[E\left(\int_{0}^{t}\left\|f_{2}(s)\right\|^{p} d s\right)\right]^{1 / p} \\
&<\infty,
\end{aligned}
$$

i.e. $a f_{1}+b f_{2} \in S^{p}(F)$, the proof is completed.

Let $\mathcal{L}^{p}\left(\mathbf{K}\left(R^{d}\right)\right)$ denote the set of all $\mathcal{L}^{p}$-bounded progressively measurable $\mathbf{K}\left(R^{d}\right)$-valued stochastic process. Similarly, we have notations $\mathcal{L}^{p}\left(\mathbf{K}_{c}\left(R^{d}\right)\right)$, $\mathcal{L}^{p}\left(\mathbf{K}_{k}\left(R^{d}\right)\right)$ and $\mathcal{L}^{p}\left(\mathbf{K}_{k c}\left(R^{d}\right)\right)$.

Take $F_{i}=\left\{F_{i}(t): t \in I\right\} \in \mathcal{L}^{p}\left(\mathbf{K}\left(R^{d}\right)\right), i=1,2$. Since for any $t \in I$,

$$
\begin{aligned}
d_{H}\left(F_{1}(t, \omega), F_{2}(t, \omega)\right) & \leq\left\|F_{1}(t, \omega)\right\|_{\mathbf{K}}+\left\|F_{2}(t, \omega)\right\|_{\mathbf{K}} \\
& <\infty,
\end{aligned}
$$

the real-valued stochastic process $\left\{d_{H}\left(F_{1}(t), F_{2}(t)\right)\right.$ : $t \in I\}$ belongs to $\mathcal{L}^{p}(R)$. Thus, define

$$
\Delta_{p}\left(F_{1}, F_{2}\right)=\left[E\left(\int_{0}^{T} d_{H}^{p}\left(F_{1}(s, \omega), F_{2}(s, \omega)\right) d s\right)\right]^{1 / p} .
$$

$F_{1}$ and $F_{2}$ are said to be equivalent, if for any $t \in$ $I, \Delta_{p}\left(F_{1}, F_{2}\right)=0$, denoted by $F_{1}=F_{2}$. Then $\Delta_{p}$ is a metric on $\mathcal{L}^{p}\left(\mathbf{K}\left(R^{d}\right)\right)$. In fact, for any $F_{i} \in$ $\mathcal{L}^{p}\left(\mathbf{K}\left(R^{d}\right)\right), i=1,2,3$, by Minkowski inequality, we have that

$$
\begin{aligned}
& \Delta_{p}\left(F_{1}, F_{2}\right) \\
&=\left[E\left(\int_{0}^{T} d_{H}^{p}\left(F_{1}(s, \omega), F_{2}(s, \omega)\right) d s\right)\right]^{1 / p} \\
& \leq\left\{E \left[\left(\int _ { 0 } ^ { T } \left(d_{H}\left(F_{1}(s, \omega), F_{3}(s, \omega)\right)+\right.\right.\right.\right. \\
&\left.\left.\left.\left.d_{H}\left(F_{3}(s, \omega), F_{2}(s, \omega)\right)\right)^{p} d s\right)\right]\right\}^{1 / p} \\
& \leq {\left[E\left(\int_{0}^{T} d_{H}^{p}\left(F_{1}(s, \omega), F_{3}(s, \omega)\right) d s\right)\right]^{1 / p}+} \\
& {\left[E\left(\int_{0}^{T} d_{H}^{p}\left(F_{3}(s, \omega), F_{2}(s, \omega)\right) d s\right)\right]^{1 / p} } \\
&= \Delta_{p}\left(F_{1}, F_{3}\right)+\Delta_{p}\left(F_{3}, F_{2}\right) .
\end{aligned}
$$

It is easy to prove that $\left(\mathcal{L}^{p}\left(\mathbf{K}\left(R^{d}\right)\right), \Delta_{p}\right)$ is complete, $\mathcal{L}^{p}\left(\mathbf{K}_{c}\left(R^{d}\right)\right), \mathcal{L}^{p}\left(\mathbf{K}_{k}\left(R^{d}\right)\right)$ and $\mathcal{L}^{p}\left(\mathbf{K}_{k c}\left(R^{d}\right)\right)$ are closed subsets of $\left(\mathcal{L}^{p}\left(\mathbf{K}\left(R^{d}\right)\right), \Delta_{p}\right)$. Denote

$$
\||| F \mid\|_{p}=\Delta_{p}(F,\{0\})=\left[E\left(\int_{0}^{T}\|F(s)\|_{\mathbf{K}}^{p} d s\right)\right]^{1 / p} .
$$

Definition 2.10 A non-empty set $\Gamma \subset \mathcal{L}^{p}\left(R^{d}\right)$ of $R^{d}$-valued progressively measurable stochastic processes is called decomposable with respect to the progressively measurable $\sigma$-field $\mathcal{C}$, if for any $f, g \in$ $\Gamma$, any $U \in \mathcal{C}$, we have $I_{U} f+I_{U^{c}} g \in \Gamma$.

Firstly, we know that for any progressively measurable stochastic process $F \in \mathcal{L}^{p}\left(\mathbf{K}\left(R^{d}\right)\right), S^{p}(F)$ is decomposable with respect to $\sigma$-field $\mathcal{C}$. Furthermore we have the following Theorem.

Theorem 2.11 Assume that $\Gamma \subset \mathcal{L}^{p}\left(R^{d}\right)$ is a nonempty closed set of $R^{d}$-valued progressively measurable stochastic processes, then $\Gamma$ is decomposable with respect to progressively measurable $\sigma$-field $\mathcal{C}$ if and only if there exists a progressively measurable set-valued stochastic process $F \in \mathcal{L}^{p}\left(\mathbf{K}\left(R^{d}\right)\right)$ such that $\Gamma=S^{p}(F)$. Furthermore, $\Gamma$ is convex if and only if $F \in \mathcal{L}^{p}\left(\mathbf{K}_{c}\left(R^{d}\right)\right)$.

Proof If there exists a progressively measurable setvalued stochastic process $F \in \mathcal{L}^{p}\left(\mathbf{K}\left(R^{d}\right)\right)$ such that $\Gamma=S^{p}(F)$, then $\Gamma$ is decomposable with respect to $\sigma$-field $\mathcal{C}$ since $S^{p}(F)$ is decomposable, i.e. for any $f_{1}, f_{2} \in S^{p}(F), U \in \mathcal{C}$ we have $I_{U} f_{1}+I_{U^{c}} f_{2} \in \Gamma$.

Now let $\Gamma$ be a non-empty closed set of $R^{d}$ valued progressively measurable stochastic processes in $\mathcal{L}^{p}\left(R^{d}\right)$, and $\Gamma$ is decomposable with respect to $\sigma$-field $\mathcal{C}$, then there exists a sequence of 
$\left\{f_{i}: i \in N\right\} \subset \mathcal{L}^{p}\left(R^{d}\right)$ such that for any $t \in I, \omega \in$ $\Omega,\left\{f_{i}(t, \omega): i \in N\right\}$ is dense in $R^{d}$.

For every $i$, let

$$
\alpha_{i}=\inf \left\{\left\|\left|f_{i}-g\right|\right\|_{p}: g \in \Gamma\right\},
$$

then there exists a sequence $\left\{g_{i j}: j \geq 1\right\} \subset \Gamma$, such that

$$
\lim _{j \rightarrow \infty}||\left|f_{i}-g_{i j}\right| \|_{p}=\alpha_{i}
$$

Define

$$
F(t, \omega)=\operatorname{cl}\left\{g_{i j}(t, \omega): i, j \in N\right\},(t, \omega) \in I \times \Omega,
$$

then $F=\{F(t): t \in I\} \in \mathcal{L}^{p}\left(\mathbf{K}\left(R^{d}\right)\right)$.

Next we will prove $\Gamma=S^{p}(F)$. For any $f \in$ $S^{p}(F), \varepsilon>0$, by Theorem 2.7 , there exists a finite $\mathcal{C}$-measurable partition $\left\{A_{1}, \cdots, A_{n}\right\}$ of $I \times \Omega$ and $\left\{h_{1}, \cdots, h_{n}\right\} \subset\left\{g_{i j}\right\}$ such that

$$
\left\|\left|f-\sum_{k=1}^{n} I_{A_{k}} h_{k}\right|\right\|_{p}<\varepsilon .
$$

Since $\sum_{k=1}^{n} I_{A_{k}} h_{k} \in \Gamma$ and $\Gamma$ is a closed set, $f \in \Gamma$. So $S^{p}(F) \subseteq \Gamma$.

Suppose that $S^{p}(F) \varsubsetneqq \Gamma$. Then there exist $f \in \Gamma$, $A \in \mathcal{C}$, and $\delta>0$ such that

$$
\inf _{i, j}\left\|f(t, \omega)-g_{i j}(t, \omega)\right\| \geq \delta, \quad \forall(t, \omega) \in A
$$

and $(\lambda \times \mu)(A)>0$. Thus, there exists $f_{i}$ such that

$$
B=A \cap\left\{(t, \omega) \in I \times \Omega:\left\|f(t, \omega)-f_{i}(t, \omega)\right\| \leq \frac{\delta}{3}\right\}
$$

has a positive measure, and let

$$
g_{j}^{\prime}=I_{B} f+I_{\Omega \backslash B} g_{i j}, \quad j \in N,
$$

then $\left\{g_{j}^{\prime}\right\} \subset \Gamma$ with $\left\|\left|f_{i}-g_{j}^{\prime}\right|\right\|_{p} \geq \alpha_{i} \geq 0$. Since

$$
\begin{aligned}
& \left\|f_{i}(t, \omega)-g_{i j}(t, \omega)\right\| \\
& \geq\left\|f(t, \omega)-g_{i j}(t, \omega)\right\|-\left\|f(t, \omega)-f_{i}(t, \omega)\right\| \\
& \geq \frac{2 \delta}{3}
\end{aligned}
$$

$$
\begin{aligned}
\left\|\left|f_{i}-g_{i j}\right|\right\|_{p}-\alpha_{i} & \geq\left\|\left|f_{i}-g_{i j}\right|\right\|_{p}-\left\|\left|f_{i}-g_{j}^{\prime}\right|\right\|_{p} \\
& \geq T^{\frac{1}{p}}\left(\frac{2 \delta}{3}-\frac{\delta}{3}\right) \mu(B)>0 .
\end{aligned}
$$

It contracts $\left\|\left|f_{i}-g_{i j}\right|\right\|_{p} \rightarrow \alpha_{i}$ when $j \longrightarrow \infty$. Then we have $\Gamma=S^{p}(F)$.

Suppose that $\Gamma$ is convex, and $\Gamma=S^{p}(F)$. Let $G=\overline{\mathrm{co}} F$, then $S^{p}(G)$ is a closed convex set in $\mathcal{L}^{p}\left(R^{d}\right)$. Since $S^{p}(F) \subset S^{p}(G), \overline{\operatorname{co}} S^{p}(F) \subset S^{p}(G)$. Next we will prove $\overline{c o} S^{p}(F) \supset S^{p}(G)$. By Theorem 2.5 , there exists a sequence $\left\{f_{i}\right\} \subset S^{p}(F)$ such that $F(t, \omega)=\operatorname{cl}\left\{f_{i}(t, \omega), i \geq 1\right\}$, let

$$
W=\left\{g: g=\sum_{i=1}^{m} \alpha_{i} f_{i}, \alpha_{i} \in Q^{+}, \sum_{i=1}^{m} \alpha_{i}=1, m \geq 1\right\}
$$

then $W$ is a subset of $S^{p}(G)$, and $G(t, \omega)=$ $\operatorname{cl}\{g(t, \omega): g \in W\}$. For any $f \in S^{p}(G), \varepsilon>0$, by Theorem 2.7, there exist a finite measurable partition $\left\{A_{1}, \cdots, A_{n}\right\}$ of $I \times \Omega$ and stochastic processes $g_{1}, \cdots, g_{n}$ in $W$ such that

$$
\left\|\left|f-\sum_{k=1}^{n} I_{A_{k}} g_{k}\right|\right\|_{p}<\varepsilon .
$$

Thus there exists an integer $m$, for $1 \leq k \leq n$, we have $g_{k}=\sum_{i=1}^{m} \alpha_{k_{i}} f_{i}$, where $\alpha_{k_{i}} \geq 0$ and $\sum_{i=1}^{m} \alpha_{k_{i}}=1$. Therefore

$$
\sum_{k=1}^{n} I_{A_{k}} g_{k}=\sum_{k=1}^{n} I_{A_{k}}\left(\sum_{i=1}^{m} \alpha_{k_{i}} f_{i}\right)=\sum_{k=1}^{n} \sum_{i=1}^{m} \alpha_{k_{i}} I_{A_{k}} f_{i}
$$

Since $I_{A_{k}} f_{i} \in S^{p}(F), \sum_{k=1}^{n} I_{A_{k}} g_{k}$ is a convex combination of the elements in $S^{p}(F)$, which means $f \in$ $\overline{c o} S^{p}(F)$. Thus, we have $\overline{c o} S^{p}(F)=S^{p}(G)$. Hence, $F$ is convex. The opposite part is obvious, which completes the proof of Theorem.

\section{The Lebesgue integral of set-valued stochastic process.}

Definition 3.1 Let a set-valued stochastic process $F=\{F(t): t \in I\} \in \mathcal{L}^{p}\left(\mathbf{K}\left(R^{d}\right)\right), 1 \leq p<+\infty$. For any $\omega \in \Omega, t \in I$, define

$$
\text { (A) } \int_{0}^{t} F(s, \omega) d s:=\left\{\int_{0}^{t} f(s, \omega) d s: f \in S^{p}(F)\right\} \text {, }
$$

where $\int_{0}^{t} f(s, \omega) d s$ is the Lebesgue integral, (A) $\int_{0}^{t} F(s, \omega) d s$ is called the Aumann type Lebesgue integral of set-valued stochastic process $F$ with respect to time $t$. For any $0 \leq u<t<\infty$,

$$
\text { (A) } \int_{u}^{t} F(s, \omega) d s:=(A) \int_{0}^{t} I_{[u, t]}(s) F(s, \omega) d s .
$$


Remark 3.2 In the definition 3.1, the set of selections is $S^{p}(F), 1 \leq p<+\infty$. As a matter of fact, if we only consider the Lebesgue integral, we can use $S^{1}(F)$. But we often consider the sum of integral a set-valued stochastic process with respect to time $t$ and integral of a set-valued stochastic process with respect to Brown motion, where we have to use $S^{2}(F)$. Thus we here use $S^{p}(F)$ for more general case.

Theorem 3.3 Let a set-valued stochastic process $F=\{F(t): t \in I\} \in \mathcal{L}^{p}\left(\mathbf{K}\left(R^{d}\right)\right)$, then for any $t \in I$, (A) $\int_{0}^{t} F(s) d s$ is a non-empty subset of $L^{p}\left[\Omega, \mathcal{A}_{t}, \mu ; R^{d}\right]$. Furthermore, if $F \in \mathcal{L}^{p}\left(\mathbf{K}_{c}\left(R^{d}\right)\right)$, then for any $t \in I,(A) \int_{0}^{t} F(s) d s$ is a non-empty convex subset of $L^{p}\left[\Omega, \mathcal{A}_{t}, \mu ; R^{d}\right]$.

Proof Since $S^{p}(F)$ is non-empty and by the Jensen inequality of integral, it is easy to know that $(A) \int_{0}^{t} F(s) d s$ is a non-empty subset of $L^{p}\left[\Omega, \mathcal{A}_{t}, \mu ; R^{d}\right]$. If $F \in \mathcal{L}^{p}\left(\mathbf{K}_{c}\left(R^{d}\right)\right)$, then by Theorem $2.9, S^{p}(F)$ is a convex subset of $\mathcal{L}^{p}\left(R^{d}\right)$. Thus (A) $\int_{0}^{t} F(s) d s$ is convex.

Remark 3.4 For any $t>0$, it is natural to hope that the result of integral is a set-valued stochastic process taking values in $\mathbf{K}\left(R^{d}\right)$ rather than in $L^{p}\left[\Omega, \mathcal{A}_{t}, \mu ; R^{d}\right]$. So it is necessary to give a new definition. However, $(A) \int_{0}^{t} F(s) d s$ is not decomposable in general. Hence, we firstly give the definition of decomposable closure.

Definition 3.5 For any non-empty subset $\Gamma \subset L^{p}[I \times$ $\left.\left.\Omega, \mathcal{C}, \lambda \times \mu ; R^{d}\right)\right]$, define the decomposable closure of $\Gamma$ with respect to $\mathcal{C}$

$\overline{d e} \Gamma=\{g=\{g(t, \omega): t \in I\}$ : for any $\varepsilon>0$, there

exists a $\mathcal{C}$-measurable finite partition

$$
\left\{A_{1}, \cdots, A_{n}\right\} \text { of } I \times \Omega \text { and } f_{1}, \cdots, f_{n} \in \Gamma
$$

such that $\left.\left\|\mid g-\sum_{i=1}^{n} I_{A_{i}} f_{i}\right\|_{p}<\varepsilon\right\}$.

Theorem 3.6 Let $F=\{F(t): t \in I\} \in \mathcal{L}^{p}\left(\mathbf{K}\left(R^{d}\right)\right)$, $\Gamma(t)=(A) \int_{0}^{t} F(s) d s$, then there exists a $\mathcal{C}$ measurable set-valued stochastic process $L(F)=$ $\left\{L_{t}(F): t \in I\right\} \in \mathcal{L}^{p}\left(\mathbf{K}\left(R^{d}\right)\right)$ such that $S^{p}(L(F))=$ $\overline{d e}\{\Gamma(t): t \in I\}$. Furthermore, if $F \in \mathcal{L}^{p}\left(\mathbf{K}_{c}\left(R^{d}\right)\right)$, then $\left\{L_{t}(F): t \in I\right\} \in \mathcal{L}^{p}\left(\mathbf{K}_{c}\left(R^{d}\right)\right)$.

Proof For any $t \in I, \Gamma(t)$ is a non-empty subset of $L^{p}\left[\Omega, \mathcal{A}_{t}, \mu ; R^{d}\right]$ from Theorem 3.3. For any $x(t) \in$ $\Gamma(t)$, there exists $f \in S^{p}(F)$ such that $x(t)(\omega)=$

$$
\begin{aligned}
\int_{0}^{t} f(s, \omega) d s, & \forall \omega \in \Omega . \text { Let } \\
M= & \overline{d e}\{\Gamma(t): t \in I\} \\
= & \overline{d e}\{g=\{g(t): t \in I\}: g(t)(\omega)= \\
& \left.\int_{0}^{t} f(s, \omega) d s, f \in S^{p}(F)\right\},
\end{aligned}
$$

then $M$ is a closed convex subset of $L^{p}[I \times \Omega, \bigodot, \lambda \times$ $\left.\mu ; R^{d}\right]$ and it is decomposable with respect to $\mathcal{C}$. By Theorem 2.11, there exists a set-valued stochastic process $L(F)=\left\{L_{t}(F): t \in I\right\} \in \mathcal{L}^{p}\left(\mathbf{K}\left(R^{d}\right)\right)$ such that $S^{p}(L(F))=M$

If $F \in \mathcal{L}^{p}\left(\mathbf{K}_{c}\left(R^{d}\right)\right)$, then $\Gamma(t)$ is convex by Theorem 3.3. To finish the proof of Theorem, we only need to prove that $M=\overline{d e}\{\Gamma(t): t \in I\}$ is convex from Theorem 2.11.

For any $\phi, \psi \in M$, any $\varepsilon>0$, there exists two C- measurable partitions $\left\{A_{i}: i=1,2, \ldots, n\right\},\left\{B_{j}\right.$ : $j=1,2, \ldots, m\}$ of $I \times \Omega$ and $\left\{\phi_{i}: i=1,2, \ldots, n\right\}$, $\left\{\psi_{j}: j=1,2, \ldots, m\right\} \subset \mathrm{U}:=\{g=\{g(t): t \in I\}:$ $\left.g(t)=\int_{0}^{t} f(s) d s,\{f(\cdot)\} \in S^{p}(F)\right\}$ such that

$$
\begin{gathered}
\left\|\mid \phi-\sum_{i=1}^{n} I_{A_{i}} \phi_{i}\right\| \|_{p}<\varepsilon, \\
\left\|\mid \psi-\sum_{j=1}^{m} I_{B_{j}} \psi_{j}\right\| \|_{p}<\varepsilon .
\end{gathered}
$$

For any $\alpha \in[0,1]$, we have that

$$
\begin{aligned}
& \left\|\alpha \phi+(1-\alpha) \psi-\alpha \sum_{i=1}^{n} I_{A_{i}} \phi_{i}-(1-\alpha) \sum_{j=1}^{m} I_{B_{j}} \psi_{j} \mid\right\|_{p} \\
& \leq \alpha\left|\left\|\phi-\sum_{i=1}^{n} I_{A_{i}} \phi_{i}\left|\left\|_{p}+(1-\alpha)\right\|\left\|\psi-\sum_{j=1}^{m} I_{B_{j}} \psi_{j} \mid\right\|_{p}\right.\right.\right. \\
& \leq \alpha \varepsilon+(1-\alpha) \varepsilon=\varepsilon,
\end{aligned}
$$

and

$$
\begin{aligned}
& \alpha \sum_{i=1}^{n} I_{A_{i}} \phi_{i}+(1-\alpha) \sum_{j=1}^{m} I_{B_{j}} \psi_{j} \\
& =\sum_{i=1}^{n} \sum_{j=1}^{m} I_{A_{i} \cap B_{j}}\left(\alpha \phi_{i}+(1-\alpha) \psi_{j}\right) .
\end{aligned}
$$

Since $S^{p}(F)$ is convex, $\left\{\alpha \phi_{i}+(1-\alpha) \psi_{j}: i=\right.$ $1, \cdots, n ; j=1, \cdots, m\} \subset U$. This with $\left\{A_{i} \cap B_{j}\right.$ : $i=1, \cdots, n ; j=1, \cdots, m\}$ being also a $\mathcal{C}$-measurable partition of $I \times \Omega$ implies $\alpha \phi+(1-\alpha) \psi \in \overline{d e} U=M$, the proof is completed. 
Remark 3.7 We can prove that the decomposable closure of $\Gamma(t)=(A) \int_{0}^{t} F(s) d s$ is bounded in $\mathcal{L}^{p}$. That is,

$$
\begin{aligned}
& \left\|\mid \sum_{i=1}^{n} I_{A_{i}} \int_{0}^{t} f_{i}(s, \omega) d s\right\|_{p} \\
& =\left[E \int_{0}^{T}\left\|\sum_{i=1}^{n} I_{A_{i}} \int_{0}^{t} f_{i}(s, \omega) d s\right\|^{p} d t\right]^{\frac{1}{p}} \\
& \leq\left[E \int_{0}^{T}\left(\sum_{i=1}^{n}\left\|I_{A_{i}} \int_{0}^{t} f_{i}(s, \omega) d s\right\|\right)^{p} d t\right]^{\frac{1}{p}} \\
& \leq\left[E \int_{0}^{T}\left(\sum_{i=1}^{n}\left\|I_{A_{i}}\right\|\left\|\int_{0}^{t} f_{i}(s, \omega) d s\right\|\right)^{p} d t\right]^{\frac{1}{p}} \\
& \leq\left[E \int_{0}^{T}\left(\sum_{i=1}^{n} I_{A_{i}} \int_{0}^{t}\left\|f_{i}(s, \omega)\right\| d s\right)^{p} d t\right]^{\frac{1}{p}} \\
& \leq\left[E \int_{0}^{T}\left(\sum_{i=1}^{n} I_{A_{i}} \int_{0}^{t}\|F(s, \omega)\| \mathbf{K} d s\right)^{p} d t\right]^{\frac{1}{p}} \\
& \leq\left[E \int_{0}^{T}\left(\sum_{i=1}^{n} I_{A_{i}} \int_{0}^{T}\|F(s, \omega)\|_{\mathbf{K}} d s\right)^{p} d t\right]^{\frac{1}{p}} \\
& =\left[E \int_{0}^{T}\left(\int_{0}^{T}\|F(s, \omega)\|_{\mathbf{K}} d s\right)^{p}\left(\sum_{i=1}^{n} I_{A_{i}}(t)\right)^{p} d t\right]^{\frac{1}{p}} \\
& =\left[E \int_{0}^{T}\left(\int_{0}^{T}\|F(s, \omega)\|_{\mathbf{K}} d s\right)^{p} d t\right]^{\frac{1}{p}} \\
& <C
\end{aligned}
$$

where $C$ is a constant and is not relative to $n$.

Definition 3.8 The set-valued stochastic process $L(F)=\left\{L_{t}(F): t \in I\right\}$ defined in Theorem 3.6 is called the Lebesgue integral of a set-valued stochastic process $F=\left\{F_{t}: t \in I\right\} \in \mathcal{L}_{T}^{p}\left(\mathbf{K}\left(R^{d}\right)\right)$ with respect to the time $t$, and denoted as $L_{t}(F)=$ (L) $\int_{0}^{t} F(s) d s$.

Now we state the representation theorem of Lebesgue integral of the set-valued stochastic process.

Theorem 3.9 Let $F=\left\{F_{t}: t \in I\right\} \in \mathcal{L}^{p}\left(\mathbf{K}\left(R^{d}\right)\right)$, then there exists a sequence of $R^{d}$-valued stochastic processes $\left\{f^{i}=\left\{f^{i}(t): t \in I\right\}: i \geq 1\right\} \subset S^{p}(F)$ such that

$$
F(t, \omega)=\operatorname{cl}\left\{f^{i}(t, \omega): i \geq 1\right\}, \quad \text { a.e. }(t, \omega) \in I \times \Omega,
$$

and

$$
L_{t}(F)=\operatorname{cl}\left\{\int_{0}^{t} f^{i}(s, \omega) d s: i \geq 1\right\} \quad \text { a.e. }(t, \omega) \in I \times \Omega .
$$

Proof For any $t \in I,\left\{L_{t}(F): t \in I\right\} \in \mathcal{L}^{p}\left(\mathbf{K}\left(R^{d}\right)\right)$ from Theorem 3.6. By virtue of Theorem 2.5, there exists a sequence of $\left\{\phi_{n}=\left\{\phi_{n}(t): t \in I\right\}: n \geq 1\right\} \subset$ $S^{p}(L(F))$ such that

$$
L_{t}(F)(\omega)=\operatorname{cl}\left\{\phi_{n}(t, \omega): n \geq 1\right\} \text {, a.e. }(t, \omega) \in I \times \Omega .
$$

Since

$$
\begin{aligned}
S^{p}(L(F)) & \overline{d e}\{\Gamma(t): t \in I\} \\
= & \overline{d e}\left\{g=\{g(t): t \in I\}: g(t)=\int_{0}^{t} f(s) d s,\right. \\
& \left.\{f(\cdot)\} \in S^{p}(F)\right\} \\
= & \operatorname{cl}\left\{h=\{h(t): t \in I\}: h(t)=\sum_{k=1}^{l} I_{A_{k}} \int_{0}^{t} f_{k}(s) d s,\right. \\
& \left\{A_{k}: k=1.2, \cdots, l\right\} \subset \mathcal{C} \text { is a finite partition of } \\
& I \times \Omega \text { and }\left\{\left\{f_{k}(\cdot)\right\}: k=1, \cdots, l\right\} \subset S^{p}(F), \\
& l \geq 1\},
\end{aligned}
$$

then for any $n \geq 1$, there exists $\left\{h_{n}^{i}: i \geq 1\right\}$ such that $\left\|\mid \phi_{n}(t)-h_{n}^{i}(t)\right\| \|_{p} \rightarrow 0 \quad(i \rightarrow \infty)$, and

$$
h_{n}^{i}(t)=\sum_{k=1}^{l(i, n)} I_{A_{k}^{(i, n)}} \int_{0}^{t} f_{k}^{(i, n)}(s) d s,
$$

where $\left\{A_{k}^{(i, n)}: k=1.2, \cdots, l(i, n)\right\} \subset \mathcal{C}$ is a finite partition of $\Omega$ and $\left\{\left\{f_{k}^{(i, n)}(t): t \in I\right\}: k=\right.$ $1,2, \cdots, l(i, n)\} \subset S^{p}(F)$. Hence, there exists a subsequence $\left\{i_{j}: j \geq 1\right\}$ of $\{1,2, \cdots\}$ such that $\left\|\phi_{n}(t, \omega)-h_{n}^{i_{j}}(t, \omega)\right\|^{p} \rightarrow 0$ a.e. $(j \rightarrow \infty)$. Thus, for a.e. $(t, \omega) \in I \times \Omega$, we have that

$$
\begin{aligned}
L_{t}(F)(\omega)= & \operatorname{cl}\left\{h_{n}^{i_{j}}(t, \omega): n, j \geq 1\right\} \\
\subset & \operatorname{cl}\left\{\int_{0}^{t} f_{k}^{\left(i_{j}, n\right)}(s, \omega) d s: n, j \geq 1,\right. \\
& \left.k=1, \cdots, l\left(i_{j}, n\right)\right\} \\
\subset & L_{t}(F)(\omega) .
\end{aligned}
$$

This implies that for a.e. $(t, \omega) \in I \times \Omega$, we have

$$
\begin{gathered}
L_{t}(F)(\omega)=\operatorname{cl}\left\{\int_{0}^{t} f_{k}^{\left(i_{j}, n\right)}(s, \omega) d s: n, j \geq 1,\right. \\
\left.k=1, \cdots, l\left(i_{j}, n\right)\right\} .
\end{gathered}
$$


Since $F \in \mathcal{L}^{p}\left(\mathbf{K}\left(R^{d}\right)\right)$ and from Theorem 2.5, there exists a sequence $\left\{\xi_{d}(t): t \in I\right\} \in S^{p}(F), d \geq 1$ such that for any $(t, \omega) \in I \times \Omega$,

$$
F(t, \omega)=\operatorname{cl}\left\{\xi_{d}(t, \omega): d \geq 1\right\} .
$$

We rewrite the countable set $\left\{\left\{\xi_{d}(t): t \in\right.\right.$ $\left.I\},\left\{f_{k}^{\left(i_{j}, n\right)}(t): t \in I\right\}: n, j, d \geq 1, k=1, \cdots, l\left(i_{j}, n\right)\right\}$, as $\left\{\left\{f^{i}(t): t \in I\right\}: i \geq 1\right\}$. Then from (3.1) and (3.2), $\left\{\left\{f^{i}(t): t \in I\right\}: i \geq 1\right\}$ satisfies the Theorem. The proof is completed.

Example 3.10 Let $f=\left\{f_{t}: t \in I\right\}, g=\left\{g_{t}: t \in I\right\}$ be real valued stochastic processes satisfying that for a.e. $(t, \omega) \in I \times \Omega, f(t, \omega)<g(t, \omega)$, and $f, g \in$ $\mathcal{L}^{p}(R)$. Define $F_{t}(\omega)=\left[f_{t}(\omega), g_{t}(\omega)\right]$, then it is easy to have that $F=\left\{F_{t}, \mathcal{A}_{t}: t \geq 0\right\}$ is a $L^{p}$-bounded setvalued stochastic process.

If $h=\left\{h_{t}: t \in I\right\} \in \mathcal{L}^{p}(R)$ satisfying $f_{t}(\omega) \leq$ $h_{t}(\omega) \leq g_{t}(\omega)$ for a.e. $(t, \omega) \in I \times \Omega$, then $h \in S^{p}(F)$ and $S^{p}(F)=\left\{h=\left\{h_{t}: t \in I\right\} \in \mathcal{L}^{p}(R): f_{t}(\omega) \leq\right.$ $h_{t}(\omega) \leq g_{t}(\omega)$ for a.e. $\left.(t, \omega) \in I \times \Omega\right\}$. Thus, there exists a sequence of real valued stochastic process $\left\{h^{i}=\left\{h^{i}(t): t \in I\right\}: i \geq 1\right\} \subset S^{p}(F)$ such that

$$
F(t, \omega)=\operatorname{cl}\left\{h^{i}(t, \omega): i \geq 1\right\}, \quad \text { a.e. }(t, \omega) \in I \times \Omega,
$$

and

$L_{t}(F)$

$=\operatorname{cl}\left\{\int_{0}^{t} h^{i}(s, \omega) d s: i \geq 1\right\}$

$=\left[\int_{0}^{t} f(t, \omega) d s, \int_{0}^{t} g(t, \omega) d s\right]$, a.e. $(t, \omega) \in I \times \Omega$.

Theorem 3.11 Let set-valued stochastic processes $F^{(i)}=\left\{F^{(i)}(t), \mathcal{A}_{t}: t \in I\right\} \in \mathcal{L}^{p}\left(\mathbf{K}\left(R^{d}\right)\right), i=1,2$, then for any $t \in I$,

$$
\begin{aligned}
& (L) \int_{0}^{t} \operatorname{cl}\left(F^{(1)}(s, \omega)+F^{(2)}(s, \omega)\right) d s \\
& =\operatorname{cl}\left\{(L) \int_{0}^{t} F^{(1)}(s) d s+(L) \int_{0}^{t} F^{(2)}(s, \omega) d s\right\} .
\end{aligned}
$$

Proof It only needs to prove the equality

$$
\begin{aligned}
& S^{p}\left(L\left(\mathrm{cl}\left(F^{(1)}+F^{(2)}\right)\right)\right) \\
& =\operatorname{cl}\left(S^{p}\left(L\left(F^{(1)}\right)\right)+S^{p}\left(L\left(F^{(2)}\right)\right)\right) .
\end{aligned}
$$

In fact,

$$
\begin{aligned}
& S^{p}\left(L\left(\operatorname{cl}\left(F^{(1)}+F^{(2)}\right)\right)\right) \overline{\operatorname{de}}\left\{g=\{g(t): t \in I\}: g(t)=\int_{0}^{t} f(s) d s,\right. \\
&\left.\{f(\cdot)\} \in S^{p}\left(\operatorname{cl}\left(F^{(1)}+F^{(2)}\right)\right)\right\} \\
&=\overline{\operatorname{de}}\left\{g=\{g(t): t \in I\}: g(t)=\int_{0}^{t} f(s) d s,\right. \\
&=\overline{\operatorname{de}}\left\{h=\{h(t): t \in I\}: h(t):=g^{(1)}(t)+g^{(2)}(t)\right. \\
&:=\int_{0}^{t} f^{(1)}(s) d s+\int_{0}^{t} f^{(2)}(s) d s, \\
&\left.\left\{f^{(1)}(\cdot)\right\} \in S^{p}\left(F^{(1)}\right),\left\{f^{(2)}(\cdot)\right\} \in S^{p}\left(F^{(2)}\right)\right\} \\
&=\operatorname{cl}\left\{\overline { \operatorname { d e } } \left\{g=\left\{g^{(1)}(t): t \in I\right\}: g^{(1)}(t)=\right.\right. \\
&\left.\int_{0}^{t} f^{(1)}(s) d s,\left\{f^{(1)}(\cdot)\right\} \in S^{p}\left(F^{(1)}\right)\right\} \\
&+\overline{\operatorname{de}}\left\{g=\left\{g^{(2)}(t): t \in I\right\}: g^{(2)}(t)=\right. \\
&\left.\left.\int_{0}^{t} f^{(2)}(s) d s,\left\{f^{(2)}(s)\right\} \in S^{p}\left(F^{(2)}\right)\right\}\right\} \\
&=\operatorname{cl}\left(S^{p}\left(L\left(F^{(1)}\right)\right)+S^{p}\left(L\left(F^{(2)}\right)\right) .\right.
\end{aligned}
$$

\section{Conclusions}

In this paper, we firstly stated the necessities of studying set-valued stochastic integrals. It may be useful in the area of stochastic control and mathematical finance. Secondly, we defined a new type Lebesgue integral of a set-valued stochastic process with respect to time $t$ based on the nice works such as Kisielewicz ${ }^{6}$, Kim ${ }^{15}$, M. Kisielewicz, M. Michta and J. Motyl 13,14. And then we discussed some properties of set-valued Lebesgue integral, especially we proved the presentation theorem of setvalued stochastic integral. Those results will be useful to study set-valued stochastic differential equations and their applications.

\section{Acknowledgments}

We would like to thank the referees for their valuable comments that considerably improved the presentation of this paper. 
This research is supported by NSFC(No.10771010), Research fund of Beijing Educational Committee, PHR(IHLB) and 111 Talent Project Fund of BJUT, P.R. China.

\section{References}

1. B. Oksendal Stochastic Differential Equations, Springer-Verlag, 1995.

2. N. Ikeda and S. Watanabe, Stochastic Differential Equations and Diffusion Processes, Noth-Holland: Kodansha, Tokyo, 1981.

3. I. Karatzas, Lectures on the Mathematics of Finance, American Mathematical Society, Providence, Rhode Island USA, 1997.

4. N.U. Ahmed, "Nonlinear Stochastic differential inclusions on Banach space," Stoch. Anal. Appl., 12 1-10 (1994).

5. M. Kisielewicz, "Set valued stochastic integrals and stochastic inclusions," Discuss. Math., 13 119-126, (1993).

6. M. Kisielewicz, "Properties of solution set of stochastic inclusions," J. Appl. Math. Stoch. Anal., 6 217-236 ,(1993).

7. M. Kisielewicz, "Existence theorem for nonconvex stochastic inclusions," J. Appl. Math. Stoch. Anal., 7 151-159,(1994).

8. M. Kisielewicz, "Viability theorems for stochastic inclusions," Discuss. Math., 15 61-74,(1995).

9. M. Kisielewicz, "Set-valued stochastic integrals and stochastic inclusions," Stoch. Anal. Appl. 15 783-800, (1997).

10. M. Kisielewicz, "Quasi-retractive representation of solusion sets to stochastic inclusions," J. Appl. Math. Stoch. Anal., 10 227-238,(1997).

11. J.P. Aubin and G.D. Prato, "The viability theorem for stochastic differenrial inclusions, "Stoch. Anal. Allp., 16 1-15, (1998).

12. J. Motyl, "Stability problem for stochastic inclusion," Stoch. Anal. Appl., 16 933-944,(1998).

13. M. Kisielewicz, M. Michta and J. Motyl, "Set valued approach to stochastic control part I (existence and regularity properties)." Dynamic Systs and Appl., 12 405-432,(2003).

14. M. Kisielewicz, M. Michta and J. Motyl, "Set valued approach to stochastic control part II (viability and semimartingale issues)." Dynamic Systs and Appl., 12 433-466,(2003).

15. B.K. Kim and J.H. Kim, "Stochastic integrals of setvalued processes and fuzzy processes," J. Math. Anal. Appl., 236 480-502,(1999).

16. R. Aumann , "Integrals of set valued functions, " $J$. Math. Anal. Appl., 12 1-12,(1965).

17. E.J. Jung and J.H. Kim, "On set-valued stochastic integrals,” Stoch. Anal. Appl., 21 401-418, (2003).

18. S. Li and A. Ren, "Representation theorems, setvalued and fuzzy set-valued Ito intergal," Fuzzy Sets and Syst., 158 949-962,(2007).

19. S. Li, Y. Ogura and V. Kreinovich, Limit Theorems and Applications of Set-Valuded and Fuzzy SetsValued Random Variables, Kluwer Academic Publishers, 2002.

20. F. Hiai and H. Umegaki,"Integrals, conditional expectations and martingales of multivalued functions," Jour. Multivar. Anal., 7 149-182,(1977).

21. W. Zhang, S. Li, Z. Wang and Y. Gao, An Introduction of Set-Valued Stochastic Processes, Sinence Press, Beijing/New York, 2007.

22. J.P. Aubin and H. Frankowska, Set-Valued Analysis, Birkhauser, 1990.

23. G. Beer, Topologies on Closed and Closed Convex Sets, Kluwer Academic Publishers, 1993.

24. C. Castaing and M. Valadier, Convex Analysis and Measurable Multifunctions, Lect. Notes in Math., 580, Springer-Verlag, Berlin, New York, 1977.

25. Z. Chen and R. Kulperger, "Minmax pricing and Choquet pricing," Insurance: Mathematics and Economics, 38 518-528, (2006).

26. G. Da Prato and H. Frankowska, "A stochastic Filippov theorem," Stoch. Anal. Appl. 12 409-426, (1994).

27. C. Hess, "On multivalued martingales whose values may be unbounded: martingale selectors and Mosco convergence," Jour. Multivar. Anal., 39 175201, (1991).

28. F. Hiai, "Multivalued stochastic integrals and stochastic differential inclusions," (Unpublished manuscript).

29. S. Hu and N.S. Papageorgiou Handbook of Multivalued Analysis, Kluwer Academic Publishers, 1997.

30. M. Kisielewicz, "Weak compactness of solution sets to stochastic differential inclusions with nonconvex right-hand sides," Stoch. Anal. Appl., 23 871901,(2005)

31. E. Klein and A.C. Thompson, Theory of Correspondences Including Applications to Mathematical Economics, John Wiley \& Sons, 1984.

32. S. Li and L. Guan, "Decomposition and representation theorem of set-valued amarts," to appear in International Journal of Approximate Reasoning, doi:10.1016/j.ijar.2006.12.006,(2007).

33. S. Li and Y. Ogura, "Convergence of set valued sub- and super-martingales in the Kuratowski-Mosco sense," Ann. Probab., 26 1384-1402,(1998).

34. S. Li and Y. Ogura, "Convergence of set valued and fuzzy valued martingales." Fuzzy Sets and Syst., 101 453-461,(1999).

35. S. Li, Y. Ogura, F.N. Proske and M.L. Puri, "Central Limit Theorems for Generalized Set-Valued Random Variables," J. Math. Anal. Appl., 285 250-263,(2003).

36. D.Q. Luu, "Representations and regularity of multivalued martingales," Acta Math. Vietn., 6 29-40, (1981).

37. J. Motyl, "Existence of Solutions of set-valued Ito equation," Bull. Acad. Pol. Sci., 46 419-430,(1998).

38. N. S. Papageorgiou, "On the conditional expectation 
and convergence properties of random sets," Trans. Amer. Math. Soc., 347 2495-2515,(1995).

39. M.L. Puri and D.A. Ralescu, "Strong law of large numbers for Banach space valued random sets," Ann. Probab., 11 222-224,(1983).

40. M.L. Puri and D.A. Ralescu, "Differentials of fuzzy functions," J. Math. Anal. Appl. , 91 552-558,(1983).

41. M.L. Puri and D.A. Ralescu, "Fuzzy random variables," J. Math. Anal. Appl., 114 406-422,(1986).

42. L.C.G. Rogers and D. Williams, Diffusions, Markov Processes and Martingales, Volume 2: Ito Calculus, Cambridge University Press, 2000. 